\title{
Growing networks with two vertex types
}

\author{
K. Austin and G. J. Rodgers \\ Department of Mathematical Sciences, Brunel University, \\ Uxbridge, Middlesex UB8 ЗPH, U.K.
}

\begin{abstract}
Growing networks are introduced in which the vertices are allocated one of two possible growth rates; type A with probability $p(t)$, or type $\mathrm{B}$ with probability $1-p(t)$. We investigate the networks using rate equations to obtain their degree distributions. In the first model (I), the network is constructed by connecting an arriving vertex to either a type A vertex of degree $k$ with rate $\mu k$, where $\mu \geq 0$, or to a type $\mathrm{B}$ vertex of degree $k$ with rate $k$. We study several $p(t)$, starting with $p(t)$ as a constant and then considering networks where $p(t)$ depends on network parameters that change with time. We find the degree distributions to be power laws with exponents mostly in the range $2 \leq \gamma \leq 3$. In the second model (II), the network is constructed in the same way but with growth rate $k$ for type A vertices and 1 for type $\mathrm{B}$ vertices. We analyse the case $p(t)=c$, where $0 \leq c \leq 1$ is a constant, and again find a power law degree distribution with an exponent $2 \leq \gamma \leq 3$.

Keywords: Growing random networks, power-laws
\end{abstract}

PACS numbers: 02.50.cw, 05.40.-a, 89.75Hc.

Typeset using REVTEX 


\section{INTRODUCTION}

Many networks, both in nature and society, show highly organised structure and behaviour. Examples include the world-wide-web, the internet, ecosystems, neural networks and actor collaboration networks [1-5]). These complex networks are made up of many interacting components that organise themselves according to certain rules of interaction, or organising principles.

Recently, a number of growing network models have been introduced [6] in an attempt to discover the organising principles governing the behaviour of these complex networks. In particular, large numbers of the models introduce new vertices and edges to the network over time to model growth and evolution in complex networks.

The best way of adding new vertices and edges to networks is the process of preferential attachment, whereby vertices with more edges increase their degree more quickly. This process results in the power law degree distribution, often found in real growing networks. Systems that possess such power law degree distributions are found to be highly tolerant to random error and attack [7], and so are exceptionally robust. Preferential attachment naturally results in these power law degree distributions and seems to be a natural way of introducing new vertices and edges to the network. It seems reasonable that more highly connected vertices should be more likely to receive new edges. For example, experience tells us that well established internet sites are more likely to attract the attention of new sites than are their lesser connected counterparts.

Due to its success, linear preferential attachment is now incorporated into most growing network models, with a vertex of degree $k$ receiving a new edge with probability $\Pi(k)$, given by $\Pi(k) \propto k$. This results in a power law degree distribution for large $t$, where the number of vertices of degree $k$ at time $t, n_{k}(t)$ is given by $n_{k}(t) \sim t k^{-3}$ [8]. If $\Pi(k) \propto k^{\alpha}$, where $\alpha>0$ is a constant, then it is found that for $\alpha<1, n_{k}(t)$ follows a stretched exponential and for $\alpha>1$, a single vertex in the network links to all other vertices.

Recently [9], $(k)$ was measured directly for four different growing networks. This was 
achieved using a numerical method on the computerised data of the networks. The science citation network and the internet were found to have degree distributions that are consistent with preferential attachment given by $\Pi(k) \propto k$, while the degree distributions of the actor collaboration and science co-authorship networks are consistent with preferential attachment given by $\Pi(k) \propto k^{\alpha}$ with $\alpha<1$. Despite the diversity of growing networks, most real growing networks are found to have a degree distribution given by $n_{k}(t) \sim t k^{-\gamma}$, with $2 \leq \gamma \leq 3$, and so are scale-free [10]. This scale-free nature of the networks is destroyed for nonlinear preferential attachment [8], which suggests that the networks obey linear preferential attachment but also that there is some other mechanism at work.

In this paper we introduce and study a set of models in which the vertices are allocated one of two possible growth rates; type A with probability $p(t)$, or type B with probability $1-p(t)$. In our first model $(\mathrm{I})$, both types of vertex grow with preferential attachment, but at different rates. We consider several $p(t)$, starting with $p(t)$ as a constant, and then considering cases where $p(t)$ depends on the total number of edges acquired by the type A and type B vertices. In our second model (II), only the type A vertices grow with preferential attachment, while the type $\mathrm{B}$ vertices grow at a constant rate.

In the following sections we introduce our models and present results, concentrating on the shapes of the degree distributions for the different models. In the final section we summarise our results and compare them to empirical measurements on real networks.

\section{MODEL I}

The different rates with which vertices acquire edges is often attributed to the ability of vertices to compete for new edges, called the vertex fitness [11]. In our model, we assign a fitness parameter to each vertex type, causing them to grow at different rates. At each time step a new vertex and edge is added to the network and connects to a vertex already present in the network. As in [11], we assume that the probability that a new vertex will connect to an existing vertex, $\Pi_{i}(k)$, depends on the degree $k$ and the fitness $\eta_{i}$ of that vertex, such 
that

$$
\Pi_{i}(k)=\frac{\eta_{i} k+\lambda_{i}}{\sum_{j}\left(\eta_{j} k_{j}+\lambda_{j}\right)},
$$

where the $\lambda_{j}$ are constants. The vertices in our model are either type A or type B, with $\lambda_{A}=\lambda_{B}=0, \eta_{A}=\mu$, for all type $\mathrm{A}$ vertices, where $\mu \geq 0$ is a constant, and $\eta_{B}=1$ for all type $\mathrm{B}$ vertices. Introducing $a_{k}(t)$ and $b_{k}(t)$ as the number of type $\mathrm{A}$ and type $\mathrm{B}$ vertices of degree $k$ at time $t$, we define the total number of vertices of type A, $N_{A}(t)$, and type B, $N_{B}(t)$, to be

$$
N_{A}(t)=\sum_{k=1}^{\infty} a_{k}(t) \quad \text { and } \quad N_{B}(t)=\sum_{k=1}^{\infty} b_{k}(t) .
$$

Similarly we define the total degrees of the type A and type B vertices, $M_{A}(t)$ and $M_{B}(t)$, to be

$$
M_{A}(t)=\sum_{k=1}^{\infty} k a_{k}(t) \quad \text { and } \quad M_{B}(t)=\sum_{k=1}^{\infty} k b_{k}(t) .
$$

Eq. (1) then gives $\Pi_{A}(k)=\mu k / M(t)$ and $\Pi_{B}(k)=k / M(t)$ as the rates with which the new vertices will connect to a type A or type B vertex of degree $k$, where $M(t)=\mu M_{A}(t)+M_{B}(t)$. Following [8], the rate equations for $a_{k}(t)$ and $b_{k}(t)$ are

$$
\frac{d a_{k}(t)}{d t}=\frac{\mu}{M(t)}\left[(k-1) a_{k-1}-k a_{k}\right]+p(t) \delta_{k, 1}
$$

and

$$
\frac{d b_{k}(t)}{d t}=\frac{1}{M(t)}\left[(k-1) b_{k-1}-k b_{k}\right]+(1-p(t)) \delta_{k, 1} .
$$

The first term on the right hand side of these equations represents the creation of a vertex of degree $k$ from a vertex of degree $k-1$. The second term represents the creation of a vertex of degree $k+1$ from a vertex of degree $k$. This happens with rate $\mu k$ for the type A vertices and with rate $k$ for the type B vertices. The final term represents the arrival of a new vertex of degree 1 . The probability that new vertices are of type $\mathrm{A}$ is $p(t)$, and that they are of type $\mathrm{B}$ is $1-p(t)$, where $p(t)$ is either constant or depends on differing network parameters for the different models. 
Case A $p(t)=c$, where $0 \leq c \leq 1$.

We first consider the case where the type of the new vertex is chosen with constant probability. As in [8], we consider only the asymptotic regime, $t \rightarrow \infty$, and find the moments $M_{A}(t)$ and $M_{B}(t)$ to be linear in time, implying that $a_{k}(t)$ and $b_{k}(t)$ are also linear in time. Accordingly, we substitute $M_{A}(t)=m_{A} t, M_{B}(t)=m_{B} t, a_{k}(t)=\alpha_{k} t$ and $b_{k}(t)=\beta_{k} t$ everywhere in our equations. From Eq. (3), substituting $p(t)=c$ in Eqs. (4) and (5) gives

$$
\frac{d M_{A}(t)}{d t}=\frac{\mu M_{A}(t)}{M(t)}+c \quad \text { and } \quad \frac{d M_{B}(t)}{d t}=\frac{M_{B}(t)}{M(t)}+1-c,
$$

and writing $d M_{A}(t) / d t=m_{A}$ and $d M_{B}(t) / d t=m_{B}$, we get two equations for $m_{A}$ and $m_{B}$ that can be solved to reveal

$$
m_{A}=\frac{-2+\mu+c(\mu-1)+\sqrt{[2-\mu-c(\mu-1)]^{2}+8 c(\mu-1)}}{2(\mu-1)}
$$

and

$$
m_{B}=\frac{(\mu-1-c \mu+c) m_{A}+2(1-c)}{(\mu-1) m_{A}+1} .
$$

With $p(t)=c$ and $M(t)=\left(\mu m_{A}+m_{B}\right) t$, and substituting $a_{k}(t)=\alpha_{k} t$ and $b_{k}(t)=\beta_{k} t$, Eqs. (4) and (5) yield the recursion relations

$$
\alpha_{k}=\frac{\mu(k-1)}{\mu\left(m_{A}+k\right)+m_{B}} \alpha_{k-1} \quad \text { and } \quad \beta_{k}=\frac{k-1}{\mu m_{A}+m_{B}+k} \beta_{k-1}
$$

for $k>1$. We find that for large $k, \alpha_{k}$ and $\beta_{k}$ have the solutions

$$
\alpha_{k} \sim k^{-A} \quad \text { and } \quad \beta_{k} \sim k^{-B}
$$

where $A$ and $B$ are given by

$$
A=\frac{\mu m_{A}+m_{B}}{\mu}+1 \quad \text { and } \quad B=\mu m_{A}+m_{B}+1
$$

We find that the degree distribution of the network, given by $n_{k}(t)=\left(\alpha_{k}+\beta_{k}\right) t$ follows a power law, $n_{k}(t) \sim t k^{-\gamma}$, for large $k$, with $\gamma=\min (A, B)$. For $\mu=0, \gamma=(3-c)$ and the type $\mathrm{B}$ vertices dominate the degree distibution. For $\mu=1, \gamma=3$, the two types of 
vertex become indistinguishable and the model reduces to that for a single vertex type [12]. As $\mu \rightarrow \infty, \gamma \rightarrow(2+c)$ and the type A vertices dominate the degree distribution. Since $0 \leq c \leq 1$, we find that $2 \leq \gamma \leq 3$ for large $k$.

For the specific case of $p(t)=0$, there is only one type A vertex in the network as the probability that a new vertex will be of type A is zero. The new vertices are therefore always of type B. With $p(t)=0$, and following the steps above, Eqs. (4) and (5) yield two sets of recursion relations. For $2<\mu<\infty$, we obtain the recursion relations

$$
\alpha_{k}=\frac{k-1}{k+1} \alpha_{k-1} \quad \text { and } \quad \beta_{k}=\frac{k-1}{k+\mu} \beta_{k-1}
$$

for $k>1$, with solutions

$$
\alpha_{k} \sim k^{-2} \quad \text { and } \quad \beta_{k} \sim k^{-(\mu+1)}
$$

for large $k$, and for $0<\mu<2$, we obtain

$$
\alpha_{k}=\frac{\mu(k-1)}{\mu k+2} \alpha_{k-1} \quad \text { and } \quad \beta_{k}=\frac{k-1}{k+2} \beta_{k-1}
$$

for $k>1$, with solutions

$$
\alpha_{k} \sim k^{-\left(\frac{2+\mu}{\mu}\right)} \quad \text { and } \quad \beta_{k} \sim k^{-3}
$$

for large $k$.

As before, the degree distribution follows a power law, $n_{k}(t)=t k^{-\gamma}$, where $2 \leq \gamma \leq 3$ for large $k$. For $\mu=0, \gamma=3$ and the type $\mathrm{B}$ vertices dominate the degree distribution. The single type A vertex, however, grows at a rate $M_{A} \sim t^{\mu / 2}$, which is generally much faster than the type $B$ vertices. As $\mu \rightarrow \infty$, the single type A vertex acquires all of the incoming edges, and grows at a rate $M_{A}(t) \sim t$. In this case, the single type $A$ vertex gains all incoming edges and the network forms a star shape.

Case B $p(t)=\mu M_{A}(t) / M(t)$.

In this case, the probabilitiy that the vertex will be of type A or type B depends on the total degree and fitness of each vertex type. If the type A vertices have higher fitness 
$(\mu>1)$, then they will grow more quickly and have a greater total degree. With both a higher fitness and a higher total degree, the new vertex will have a much higher probability of being type $\mathrm{A}$. If the type $\mathrm{B}$ vertices have higher fitness $(\mu<1)$, the new vertex will have a much higher probability of being type B. This leads to the degree distribution and the network being overwhelmingly dominated by the vertex type with the higher fitness.

Following the steps in Case A, but with $p(t)=\mu M_{A}(t) / M(t)$, Eqs. (4) and (5) yield two sets of recursion relations. For $1<\mu<\infty$, we obtain the recursion relations

$$
\alpha_{k}=\frac{k-1}{k+2} \alpha_{k-1} \quad \text { and } \quad \beta_{k}=\frac{k-1}{k+2 \mu} \beta_{k-1}
$$

for $k>1$, with solutions

$$
\alpha_{k} \sim k^{-3} \quad \text { and } \quad \beta_{k} \sim k^{-(1+2 \mu)}
$$

for large $k$, and for $0 \leq \mu<1$, we obtain the relations

$$
\alpha_{k}=\frac{\mu(k-1)}{\mu k+2} \alpha_{k-1} \quad \text { and } \quad \beta_{k}=\frac{k-1}{k+2} \beta_{k-1}
$$

for $k>1$, with solutions

$$
\alpha_{k} \sim k^{-\left(1+\frac{2}{\mu}\right)} \quad \text { and } \quad \beta_{k} \sim k^{-3}
$$

for large $\mathrm{k}$.

The degree distribution of the network again follows a power law, $n_{k}(t) \sim t k^{-\gamma}$, but with $\gamma=3$ for all $\mu \geq 0$ as $k \rightarrow \infty$. This is the behaviour we would expect, as the degree distribution is overwhelmingly dominated by just one vertex type. The effect of the second vertex type becomes negligible as $k \rightarrow \infty$ and the distribution is the same as that for just one vertex type.

Case C $p(t)=M_{B}(t) / M(t)$.

As in Case B, the new vertex type depends on the total degree and fitness of each vertex type. However, in this case, if the fitness of the type A vertices is greater, then there is a greater probability that the new vertex will be type $B$, and if the fitness of the type $B$ 
vertices is greater, there is a greater probability that the new vertex will be type A. Hence the network will have a tendancy to balance its edges out between the type A and type B vertices.

Following the method of Case A, but with $p(t)=M_{B}(t) / M(t)$, Eqs. (4) and (5) yield the recursion relations

$$
\alpha_{k}=\frac{\mu(k-1)}{\mu(k+1)+1} \alpha_{k-1} \quad \text { and } \quad \beta_{k}=\frac{k-1}{k+\mu+1} \beta_{k-1}
$$

for $k>1$, with solutions

$$
\alpha_{k} \sim k^{-\left(2+\frac{1}{\mu}\right)} \quad \text { and } \quad \beta_{k} \sim k^{-(2+\mu)}
$$

for large $k$.

We find that the vertex type with the higher fitness gains edges at an increasing rate as the difference in fitness between the two vertex types increases. This is exactly matched by the addition of an increasing number of vertices and edges of the lower fitness type so that both vertex types have a total degree of $t$ for all $\mu \geq 0$. However, the number of vertices with higher fitness added to the network decreases as the fitness gap increases, causing the vertices of this type to have a much higher degree on average. The degree distribution is then dominated by the vertex type with higher fitness, resulting in a power law degree distribution, $n_{k}(t) \sim t k^{-\gamma}$ with an exponent $\gamma$ that depends upon fitness, $\gamma=\min \left(2+\frac{1}{\mu}, 2+\mu\right)$. This gives $\gamma=2+\mu$ for $\mu<1$ and $\gamma=2+\frac{1}{\mu}$ for $\mu>1$, so that $\gamma$ is found to be in the range $2 \leq \gamma \leq 3$ for large $k$.

When the difference in fitness between the two vertex types is very large, the number of vertices of the higher fitness type is very small. The few vertices of the higher fitness type then grow with an average rate $M_{A}(a v) \sim t$ and the network develops hubs of the higher fitness type. This is analogous to the situation in Case $A$, where $p(t)=0$ and $\mu \rightarrow \infty$, where the single type A vertex acquires all of the incoming edges and the network forms a star shaped distribution.

Case D $p(t)=M_{A}(t) /\left[M_{A}(t)+M_{B}(t)\right]$. 
In this case, the type of the new vertex depends only on the total degree of each type of vertex. If the type A vertices have higher fitness, then they will grow more quickly and have a greater total degree. The new vertex will then have a greater probability of being type A. Similarly, if the type B vertices have higher fitness, the new vertex will be type B with greater probability. This is similar to Case B, as it leads to the network being dominated by the vertex type with higher fitness.

Following the steps in Case A, but with $p(t)=M_{A}(t) /\left[M_{A}(t)+M_{B}(t)\right]$, Eqs. (4) and (5) yield two sets of recursion relations. For $1<\mu<\infty$, we obtain the relations

$$
\alpha_{k}=\frac{k-1}{k+2} \alpha_{k-1} \quad \text { and } \quad \beta_{k}=\frac{k-1}{k+2 \mu} \beta_{k-1}
$$

for $k>1$, with solutions

$$
\alpha_{k} \sim k^{-3} \quad \text { and } \quad \beta_{k} \sim k^{-(2 \mu+1)}
$$

for large $k$. For $0 \leq \mu<1$ we obtain the relations

$$
\alpha_{k}=\frac{\mu(k-1)}{\mu k+2} \alpha_{k-1} \quad \text { and } \quad \beta_{k}=\frac{k-1}{k+2} \beta_{k-1}
$$

for $k>1$, with solutions

$$
\alpha_{k} \sim k^{-\left(\frac{2}{\mu}+1\right)} \quad \text { and } \quad \beta \sim k^{-3}
$$

for large $k$.

As in Case B, we find that the degree distribution of the network follows a power law, $n_{k}(t) \sim t k^{-\gamma}$ with $\gamma=3$ for all $\mu \geq 0$ as $k \rightarrow \infty$. As $k \rightarrow \infty$, the degree distribution is again dominated by just one vertex type and becomes the same distribution as that for a network with just one vertex type.

Case $\mathbf{E} p(t)=M_{B}(t) /\left[M_{A}(t)+M_{B}(t)\right]$.

As in Case D, the new vertex type depends only on the total degree of each type of vertex. In this case, if the type A vertices have higher fitness, then they will grow faster and have a greater total degree, but the new vertex will then have a greater probability 
of being type $B$. If the type $B$ vertices have higher fitness, the new vertex will be type A with greater probability. As in Case C, this has the effect of balancing out the edges of the network between the type $\mathrm{A}$ and type $\mathrm{B}$ vertices.

We again follow the steps in Case $A$, but with $p(t)=M_{B}(t) /\left[M_{A}(t)+M_{B}(t)\right]$, and get two equations that can be solved to reveal

$$
m_{A}=\frac{2(\mu-2)+2 \sqrt{\left[1-\mu+\mu^{2}\right]}}{3(\mu-1)}
$$

and

$$
m_{B}=\frac{2(1-2 \mu)+2 \sqrt{\left[1-\mu+\mu^{2}\right]}}{3(1-\mu)} .
$$

Eqs. (4) and (5) then yield the recursion relations

$$
\alpha_{k}=\frac{\mu(k-1)}{\mu\left(m_{A}+k\right)+m_{B}} \alpha_{k-1} \quad \text { and } \quad \beta_{k}=\frac{k-1}{k+\mu m_{A}+m_{B}} \beta_{k-1}
$$

for $k>1$, with solutions

$$
\alpha_{k} \sim k^{-A} \quad \text { and } \quad \beta_{k} \sim k^{-B}
$$

for large $k$, where $A$ and $B$ are given by

$$
A=\frac{\mu m_{A}+m_{B}}{\mu}+1 \quad \text { and } \quad B=\mu m_{A}+m_{B}+1
$$

As in Case $\mathrm{C}$, the vertex type with higher fitness gains edges at an increasing rate as the difference in fitness between the two vertex types increases. However, this is not exactly matched by the addition of an increasing number of vertices and edges of the lower fitness type as the gap in fitness increases. Instead, it results in a maximum total degree of $4 t / 3$ for the vertex type of higher fitness. Since the total number of edges in the network is $2 t$, the vertex type with higher fitness can only possess $1 / 2$ to $2 / 3$ of the total edges in the network. The number of vertices of each type is also limited in this case, where there is a maximum of $2 t / 3$ vertices of the lower fitness type, which is $2 / 3$ of the total vertices in the network.

The degree distribution is again dominated by the vertex type with higher fitness, resulting in a power law degree distribution, $n_{k}(t) \sim t k^{-\gamma}$, for the network with $\gamma=\min (A, B)$, 
so that $1 \leq \gamma \leq 3$ for large $k$. When the difference in fitness between the two vertex types is large, the number of vertices of the higher fitness type is $1 / 3$ of the total for the network. The vertices of the higher fitness type then grow with an average rate that is four times higher than that of the vertex type with lower fitness. With $t / 3$ vertices of much higher degree on average than the average for the network, we get a greater spread in the degree distribution, evident in the lower values obtained for the degree exponent $\gamma$.

In summary, we obtained a power law degree distribution, $n_{k}(t) \sim t k^{-\gamma}$ for all of the cases we studied in Model I. For cases $\mathrm{A}$ and $\mathrm{C}$, we found $\gamma$ to be in the range $2 \leq \gamma \leq 3$ for large $k$. For cases $\mathrm{B}$ and $\mathrm{D}$ we found that $\gamma=3$ for large $k$, and for case $\mathrm{E}$, we found that for large $k, \gamma$ is found to be in the range $1 \leq \gamma \leq 3$.

\section{MODEL II}

In the second model we consider, only one of the vertex types grows with preferential attachment, while the other grows at a constant rate. Both vertex types have a fitness of $\eta=1$, with $\lambda_{A}=0$ and $\lambda_{B}=1$, so that Eq.(1) gives $\Pi_{A}(k)=k / M(t)$ and $\Pi_{B}(k)=1 / M(t)$, where $M(t)=M_{A}(t)+N_{B}(t)$ and $N_{B}(t)$ and $M_{A}(t)$ have the definitions given in Eqs. (2) and (3). The number of vertices of type $\mathrm{A}$ and type $\mathrm{B}$ of degree $k$ at time $t, a_{k}(t)$ and $b_{k}(t)$ now obey the equations

$$
\frac{d a_{k}(t)}{d t}=\frac{1}{M(t)}\left[(k-1) a_{k-1}-k a_{k}\right]+p(t) \delta_{k, 1}
$$

and

$$
\frac{d b_{k}(t)}{d t}=\frac{1}{M(t)}\left[b_{k-1}-b_{k}\right]+(1-p(t)) \delta_{k, 1} .
$$

As before, the probability that a new vertex is of type $\mathrm{A}$ is $p(t)$, and that it is of type $\mathrm{B}$ is $1-p(t)$

We study the case where the new vertex is type A or type B with constant probability, $p(t)=c$, where $0 \leq c \leq 1$. From Eqs. (2) and (3), substituting $p(t)=c$ in Eqs. (4) and (5), gives 


$$
\frac{d M_{A}(t)}{d t}=\frac{M_{A}(t)}{M(t)}+c \quad \text { and } \quad \frac{d N_{B}(t)}{d t}=1-c
$$

and writing $d M_{A}(t) / d t=m_{A}$ and $d N_{B}(t) / d t=n_{B}$, we get two equations for $m_{A}$ and $n_{B}$ that can be solved to reveal

$$
m_{A}=c+\sqrt{c} \quad \text { and } \quad n_{B}=1-c .
$$

With $p(t)=c$ and $M(t)=(1+\sqrt{c}) t$, and writing $a_{k}(t)=\alpha t$ and $b_{k}(t)=\beta t$, Eqs. (31) and (32) yield the recursion relations

$$
\alpha_{k}=\frac{k-1}{k+\sqrt{c}+1} \alpha_{k-1} \quad \text { and } \quad \beta_{k}=\frac{1}{2+\sqrt{c}} \beta_{k-1}
$$

for $k>1$, with solutions

$$
\alpha_{k} \sim k^{-(2+\sqrt{c})} \quad \text { and } \quad \beta_{k} \sim(2+\sqrt{c})^{-k}
$$

for large $k$.

We find that the degree distribution follows a power law, $n_{k}(t) \sim t k^{-\gamma}$, where $\gamma=$ $(2+\sqrt{c})$, and since $0 \leq c \leq 1$, we find $\gamma$ to be in the range $2 \leq \gamma \leq 3$ for large $k$.

\section{DISCUSSION}

We investigated networks in which the vertices have one of two possible growth rates, type A or type B. In our first model (I), both vertex types grow with linear preferential attachment, but have different fitness, $\eta$. We analysed 5 cases, where the new vertex was type A with probability $p(t)$, and type B with probability $1-p(t)$, with different $p(t)$ in each case. We first considered the case where $p(t)$ is a constant and then went on to consider cases where $p(t)$ depends on the fitnesses and on the total degrees of the two vertex types. We found that in all the cases we studied, the degree distributions of the networks follow a power law, $n_{k}(t) \sim t k^{-\gamma}$.

For the first case, A, where $p(t)$ is constant, we found that the exponent $\gamma$ can take any value in the range $2 \leq \gamma \leq 3$, depending on the value of $p(t)$. For the specific case of 
$p(t)=0$, there is a single type A vertex that grows much faster than the type B vertices when it has higher fitness. When the single type A vertex has much higher fitness, $\mu \rightarrow \infty$, it acquires all incoming edges, monopolising the distribution. These results are inkeeping with empirical data, where most growing networks are found to have degree distributions given by $n_{k}(t) \sim t k^{-\gamma}$, with $2 \leq \gamma \leq 3$. Monopolies are also seen to occur in real systems, where in business for example, a certain company is the only supplier of a product to many consumers, or in ecology, where many parasites live off one particular host. However, although $p(t)$ is constant in our model, we are free to choose it's value in the range $0 \leq p(t) \leq 1$, so that the value of $p(t)$ can be adjusted to obtain the desired exponents for different networks.

In the two cases where the new vertex has a greater probability of being of the type with higher fitness, $\mathrm{B}$ and $\mathrm{D}$, we found that $\gamma=3$ for all fitnesses $0 \leq \mu<\infty$. The reason for this is that the contribution from the vertex type with lower fitness is negligible for large $k$, so that the network looks like a network with just one vertex type.

In the two cases where the new vertex has greater probability of being of the type with lower fitness, $\mathrm{C}$ and $\mathrm{E}$, we found that $2 \leq \gamma \leq 3$ for Case $\mathrm{C}$, when the probability is directly dependent on fitness, and $1 \leq \gamma \leq 3$ for Case $\mathrm{E}$, when it is not. In Case $\mathrm{C}$, the gain in edges of the vertex type with higher fitness is exactly balanced by the addition of more vertices and edges of the lower fitness type, so that the total degrees of the type A and type $\mathrm{B}$ vertices are equal, $M_{A}=M_{B}$. However, as the gap in fitness between the vertex types increases, less and less vertices with higher fitness are added to the network so that their average degree increases. For a very large fitness gap, this results in just the few vertices with higher fitness having a very large degree and the network again becomes monopolised by a very small number of vertices. Also, in the rare case that an incoming vertex is of the higher fitness type, it quickly overtakes many vertices already in the network, acquiring edges at a much greater rate than its lower fitness counterparts. This behaviour can be found in many networks, such as the www, where new websites can rapidly acquire links, overtaking many older websites in a short time [14].

In Case E, the gain in edges of the vertex type with higher fitness is slowed as the 
fitness gap increases, but not exactly balanced by the addition of new vertices and edges of the lower fitness type. For a very large fitness gap, the vertices of higher fitness grow with an average fitness that is much higher than that of the vertices of lower fitness. Since there can not be a very small number of vertices with higher fitness, this does not result in a few vertices monopolising the degree distribution, but results in a spread in the degree distribution, giving a power law with exponent $\gamma \sim 1$. Also, as in Case C, new vertices of higher fitness can overtake older vertices of lower fitness as observed in some real networks.

Although Model I is a simple network model and does not take into account processes such as ageing or rewiring, the model successfully incorporates a mechanism to introduce vertices of different fitness into the network. Rather than randomly adding vertices with fitnesses taken from a distribution, the fitness of the new vertex is dependent upon the state of the network at the time that it is introduced. A similar mechanism is found in networks such as ecosystems, where niches in the environment are filled by different species. As the environment changes, more of the species that are compatible with the new environment will appear. The different cases of Model I give different results, but most are consistent with empirical data.

In our second model (II), only one vertex type grows with preferential attachment, while the other grows at a constant rate. For this model, we analysed only the case where $p(t)$ is constant, and found that the degree distribution follows a power law, $n_{k}(t) \sim t k^{-\gamma}$, with

$2 \leq \gamma \leq 3$. Surprisingly, this is the same range we obtained when both vertex types grew with preferential attachment. This work suggests that the reason why most empirical measurements of real networks have power law degree distributions with exponent $2 \leq \gamma \leq 3$ is that the growth rates of vertices in these networks are inhomogeneous.

\section{ACKNOWLEDGEMENTS}

We would like to thank the Leverhulme Trust and the EPSRC for their financial support. 


\section{REFERENCES}

[1] A. Broder, R. Kumar, F. Maghoul, P. Raghavan, S. Rajagopalan, R. Stata, A. Tomkins and J. Wiener, in Proceedings of the Ninth International World Wide Web Conference, see also www.almaden.ibm.com/cs/k53/www9.final/

[2] R. Pastor-Satorras and A. Vespignani, Phys. Rev. Lett. 863200 (2001).

[3] S. A. Kauffman, The Origin of Order: Self-Organization and Selection in Evolution (Oxford University Press, London, 1993).

[4] Models of Neural Networks I, eds. E. Domany, J. L. van Hemmen, and K. Schulten, 2nd updated ed. (Springer Verlag, New York, 1995).

[5] F. Liljeros, C. R. Edling, L.A.N. Amaral, H.E. Stanley and Y. Aberg, Nature 411907 $(2001)$.

[6] R. Albert and A. L. Barabasi, Rev. Mod. Phys. 74, 47 (2002).

[7] R. Albert, H. Jeong and A. L. Barabasi, Nature 406, 378 (2000).

[8] P. L. Krapivsky, S. Redner and F. Leyvraz, Phys. Rev. Lett. 854629 (2000).

[9] H. Jeong, Z. Neda and A. L. Barabasi, Europhys Lett. 61, 567 (2003).

[10] S. N. Dorogovtsev and J. F. F. Mendes, Adv. Phys. 51, 1079 (2002).

[11] G. Bianconi and A. L. Barabasi, Europhys. Lett. 54436 (2001).

[12] P. L. Krapivsky and S. Redner, Phys. Rev. E 63066123 (2001).

[13] M. E. J. Newman, S. H. Strogatz, and D. J. Watts, Phys.Rev. E 64026118 (2001).

[14] L. A. Adamic and B. A. Huberman, Science 287, 2115 (2000). 